Exercices Exercices de rhétorique

de rhétorique $15 \mid 2020$

Sur (et contre) les figures

\title{
L'approche phénoménologique des figures de rhétorique chez Jean Paulhan
}

\section{François Demont}

\section{OpenEdition}

1 Journals

Édition électronique

URL : http://journals.openedition.org/rhetorique/1062

DOI : $10.4000 /$ rhetorique. 1062

ISSN : 2270-6909

Éditeur

UGA Éditions/Université Grenoble Alpes

Édition imprimée

ISBN : 978-2-37747-248-2

\section{Référence électronique}

François Demont, «L'approche phénoménologique des figures de rhétorique chez Jean Paulhan », Exercices de rhétorique [En ligne], 15 | 2020, mis en ligne le 07 novembre 2020, consulté le 03 décembre 2020. URL : http://journals.openedition.org/rhetorique/1062 ; DOI : https://doi.org/10.4000/ rhetorique. 1062

Ce document a été généré automatiquement le 3 décembre 2020.

\section{(c) (i) (8)}

Les contenus de la revue Exercices de rhétorique sont mis à disposition selon les termes de la Licence Creative Commons Attribution - Pas d'Utilisation Commerciale - Partage dans les Mêmes Conditions 4.0 International. 


\title{
L'approche phénoménologique des figures de rhétorique chez Jean Paulhan
}

\author{
François Demont
}

\section{Introduction : Paulhan et la réhabilitation des figures de rhétorique}

1 Dès l'entre-deux-guerres, le critique et directeur de la NRf Jean Paulhan (1884-1968) fut l'un de ceux qui, avec Paul Valéry, tentèrent d'amener la littérature moderne à se confronter à son inconscient rhétorique. Le traitement de la rhétorique, dans l'œuvre de Paulhan, est multiple ${ }^{1}$ : elle y est tantôt abordée comme discipline, tantôt comme un courant littéraire antimoderne opposé au «Terrorisme» des avant-gardes. Dans la perspective critique de Paulhan, les écrivains rhétoriqueurs revendiquent contre les écritures d'avant-garde la pratique d'une littérature informée par la rhétorique. Des auteurs comme Alain, Paul Valéry ou Julien Benda désirent, selon lui, libérer leur écriture du souci de la forme par le respect de règles pérennes et communes leur permettant de se concentrer sur le fond de leur discours. Chez eux, comme l'écrit Genette, « la parole obligée n'oblige pas² ».

2 Ainsi, dans Les Fleurs de Tarbes ou la Terreur dans les Lettres (1936/1941), Paulhan analyse notamment la relation polarisée des écrivains de sa génération à une discipline qu'ils sont plus ou moins les premiers à n'avoir pas apprise à l'école. La rhétorique en tant que discipline scolaire a en effet progressivement disparu des programmes français au profit de l'histoire littéraire entre 1885 et 1902. Paulhan remarque que ceux qu'il nomme les « Terroristes » (les dadaïstes, les surréalistes, etc.) rejettent la rhétorique, et les figures à laquelle ils la réduisent, alors qu'ils restent fascinés par elle, comme en témoigne par exemple le recyclage humoristique du lieu commun chez certains surréalistes à l'exemple d'André Breton, Benjamin Péret, René Char ou Paul Éluard ${ }^{3}$. Toutefois, il semble aux Terroristes que l'effet des figures s'est amenuisé à force de 
redites - leur haine ou leur méfiance envers les figures relevant, d'une certaine manière, d'une profonde nostalgie ou tout au moins du "remords d'une efficacité perdue ${ }^{4}$ ", celle de la parole littéraire nourrie de culture rhétorique. Autrefois fleurs de rhétorique, les figures ne sont ainsi plus considérées par les modernes que comme des fleurs de papier, même si - Langlois et Seignobos le relèvent en 1898 - « le mépris de la rhétorique, des faux brillants et des fleurs en papier n'exclut pas le goût d'un style pur et ferme, savoureux et plein $^{5}$ ». Comment, en effet, oblitérer plus de deux millénaires d'héritage rhétorique?

3 À la suite de Brunetière ${ }^{6}$, Paulhan fut ainsi l'un des principaux partisans de la revalorisation du lieu commun et de la rhétorique en France - terme qu'il faut chez lui entendre à la fois au sens de discours orné et de fonctionnement du langage. Son approche des figures est d'ailleurs entièrement polarisée par une réflexion sur le proverbe et le cliché de langue - et pas seulement de style. En effet, à l'origine du traité de Paulhan, il y a le constat d'une ambiguïté relative aux lieux communs de la rhétorique, ambiguïté qui sera ainsi décrite par Barthes : «1) ce sont des formes vides, communes à tous les arguments (plus elles sont vides, plus elles sont communes [...]);2) ce sont des stéréotypes, des propositions rabâchées ${ }^{7}$ ». Or, pour Paulhan, "les règles et les lieux communs semblent particulièrement propres à provoquer [la] dissociation du mot et de l'idée ${ }^{8} "$, de sorte que le rhétoriqueur voit dans le lieu commun le moyen le plus adapté pour traduire sa pensée alors que le Terrorise considère qu'il distord la sienne en lui imposant un moule tant utilisé qu'il empêche la communication ou la représentation de sa pensée par le langage. En cela, le lieu commun constitue le catalyseur théorique des diverses conceptions possibles du lien du langage à l'esprit qui se retrouvent dans la problématique de la figure.

Mais, plus globalement, Paulhan soutient que «toute doctrine littéraire se fonde sur une théorie du langage ${ }^{9} "$, qu'elle soit consciente et revendiquée ou non. Ainsi,

l'étude du langage et de la littérature est toujours chez Paulhan une étude des différentes attitudes que l'on peut prendre quand on traite du langage ou de la littérature. Cette approche du langage est plus phénoménologique, que linguistique ou poétique: elle est une réflexion sur les différentes phénoménalisations du langage et de la poésie. Terreur et Rhétorique sont moins des théories du langage à proprement parler que des points de vue sur le langage ${ }^{10}$.

C'est donc pour tenter de pallier une débâcle de la littérature moderne résultant d'une faille critique que Paulhan se sent l'obligation «de répondre à la crise du langage de l'entre-deux-guerres ${ }^{11} »$; ces phénomènes lui paraissant consubstantiellement liés :

Selon Paulhan, la crise du langage a une dimension à la fois politique, philosophique, esthétique. Néanmoins, elle relève d'une seule cause : la défiance vis-à-vis de l'authenticité du langage, la «misologie ». [...] Le mal du langage est donc un fait général qui dépasse la littérature mais s'y illustre de façon exemplaire ${ }^{12}$

Pour sauver la littérature et l'écriture il faudrait donc, selon Paulhan, revaloriser la rhétorique en tant que moyen permettant d'user d'une langue commune pour dire une pensée singulière. Après tout, La Bruyère a bien écrit : «Horace ou Despréaux l'a dit avant vous, je le crois sur votre parole; mais je l'ai dit comme mien, ne puis-je pas penser après eux une chose vraie, et que d'autres encore penseront après moi ${ }^{13}$ ?" Antirhétorique et héritier du romantisme, l'idéal terroriste d'une littérature qui tendrait à s'amoindrir par elle-même représente donc pour Paulhan un objet de critique et de combat essentiel. Montrer les limites et les contradictions de cet idéal pourrait, selon lui, sauver les Lettres du silence et de l'ineptie, dans un siècle dominé 
par le bavardage et le bruit. Il confie d'ailleurs dans les Fleurs de Tarbes: "Je ne sache pas de danger plus insidieux ni de malédiction plus mesquine que ceux d'un temps où maitrise et perfection désignent à peu près l'artifice et la convention vaine, où beauté, virtuosité et jusqu'à littérature signifient avant tout ce qu'il ne faut pas faire ${ }^{14}$ ». Autrement dit, de nos jours, "rien ne fait littéraire, en lettres, comme l'authentique ${ }^{15}$ ".

Or c'est étrangement dans les mêmes termes que Roland Barthes décrira lui aussi, bien des années plus tard, le discrédit où est jetée l'ancienne rhétorique. Selon lui, depuis Pascal et ses Pensées,

[...] l'éloquence consiste, non à appliquer au discours un code extérieur, mais à prendre conscience de la pensée qui naît en nous, de façon à pouvoir reproduire ce mouvement lorsque nous parlons à l'autre [...] l'ordre du discours n'a pas de caractères intrinsèques (clarté ou symétrie); il dépend de la nature de la pensée, à laquelle, pour être «droit », doit se conformer le langage ${ }^{16}$.

Car à travers les querelles littéraires sur la rhétorique, c'est bien des liens du langage à la pensée dont il est question ${ }^{17}$ alors même que la déconsidération des modernes pour la rhétorique semble mener, d'après Paulhan, à un rejet du langage lui-même. Les choses sont donc plus complexes que ce que présente Marc Fumaroli dans L'Âge de l'éloquence (1980), lorsqu'il explique que Paulhan «se prenait à réhabiliter quelque peu le vieil art des rhéteurs [et ramenait] l'attention du public lettré sur la "vieille rhétorique" 18 ". Plus qu'une simple réhabilitation, le discours paulhanien sur les figures de rhétorique révèle une critique générale portant aussi bien sur les représentations des liens du langage à la pensée que sur la littérature. Ce discours nous permet d'appréhender l'apport spécifique de Paulhan au renouveau de la rhétorique en France. L'usage théorique que fait Paulhan des figures et sa réflexion sur la relativité des étiquettes et des classements des rhétoriques anciennes configurent donc d'une part une sorte de phénoménologie herméneutique, axée sur la question de la réception du cliché - ce qui est un signe des temps, le commentaire de texte étant devenu un exercice roi dans les disciplines littéraires depuis le début du siècle. Cette sensibilité se retrouve d'ailleurs dans ses écrits sur la peinture cubiste et informelle. Et il n'est d'ailleurs pas étonnant qu'un lien fort apparaisse rétrospectivement entre les travaux de Merleau-Ponty - notamment dans La Prose du monde (1969) - et ceux de Paulhan, que Merleau-Ponty avait lus, sur le langage et l'étymologie. L'approche paulhanienne de la nature rhétorique des textes littéraires constitue, d'autre part, le ferment d'une théorie de la lecture explicitement prolongée par la théorie des textes possibles de Michel Charles $^{19}$; sa fameuse Rhétorique de la lecture (1977) est irriguée en profondeur, nous reviendrons sur ce point, par le Traité des figures. Paulhan est en effet conscient que la rhétorique est un "méta-langage (dont le langage-objet [est] le discours) ${ }^{20}$ » nécessaire à la lecture critique d'un texte littéraire.

\section{D'un Traité des figures à un Traité de phénoménologie de lecture}

6 Parmi ses nombreux écrits sur la rhétorique, Paulhan a notamment fait paraître un texte intitulé Traité des figures ou La rhétorique décryptée (1953). La chose peut paraitre intrigante, car lui-même commence son traité en prenant acte du discrédit moderne de cette discipline :

On trouve de nos jours par paquets, chez les libraires d'occasions et dans les boîtes des quais, pour un prix qui va de dix sous à trente francs, un vieux petit livre 
mystérieux. Qui nous annonce d'abord les plus beaux sujets qui soient, et les plus graves : la connaissance du langage, et des liens qui l'unissent à notre pensée [...]. Cependant, poursuit-on la lecture, ce ne sont que des règles abstruses, termes baroques, lois arbitraires, conseils trop évidents. Chaque phrase est claire, et pas une ne semble valoir la peine d'être dite. [...] Pourtant, ce petit livre a été des milliards et des milliards de fois lu, commenté, appliqué, recommencé ${ }^{21}$.

Dans un autre texte, il précise toutefois que l'« on n'a pas attendu Victor Hugo » pour critiquer la rhétorique, mais que "Platon et Montaigne", "Pascal et Diderot» s'opposaient déjà « aux habiles de leur temps et aux éloquents ${ }^{22}$ ». Malgré tout, Paulhan soutient que « la rhétorique renaît de ses cendres », et que son traité participe à cette résurgence.

Ainsi que le remarque très justement Barthes dans son texte sur l'ancienne rhétorique, "la rhétorique se donne ouvertement comme un classement (de matériaux, de règles, de parties, de genres, de styles) ${ }^{23}$ ». Ce dernier va même jusqu'à parler de "cette sorte d'activité enivrée du langage sur le langage » comme d'une «furie de découpage, de dénomination ${ }^{24} »$. Or, à l'instar des manuels antiques, le traité de Paulhan propose lui aussi un classement et un discours sur son classement - fait en soi assez habituel. Toutefois, Barthes explique :

Le classement lui-même est l'objet d'un discours: annonce du plan du traité, discussion serrée du classement proposé par les prédécesseurs. [...] la plupart du temps, et c'est normal, l'option taxinomique implique une option idéologique : il y a toujours un enjeu à la place des choses : dis-moi comment tu classes, je te dirai qui tu $e s^{25}$.

Faisant fi de la complétude des nombreux classements internes de l'elocutio des rhétoriques antiques, le traité de Paulhan se concentre explicitement sur les figures de pensées ou de mots. Il écrit ainsi : «Je n'examinerai ici que les figures ${ }^{26}$ ». Et il précise que les rhétoriques

commencent par distinguer, d'une part, les figures de langage, où l'attention se voit portée sur un mot : salut à la vousaille, voilà l'oiseau. D'autre part, les figures de pensée qui tiennent à un tour particulier de l'esprit : Comment, vous ?... Enfin, toi !... il est tout de même arrivé27.

8 Même si les figures de pensée ${ }^{28}$ ne se définissent pas par des processus formels précis et univoques, l'usage du terme "attention » sous la plume de Paulhan alors qu'il évoque les figures de langage (définies, elles, par des constructions reconnaissables formellement) est important, car il montre bien que, dans son traité, la rhétorique est abordée sous l'angle de la réception plus que de la stricte production et que la question de l'interprétation par un sujet y est essentielle. En cela, son approche se révèle en partie pré-phénoménologique, puisqu'il considère la rhétorique comme étant un outil permettant l'étude des textes et de la langue en tant qu'ils sont perçus par un sujet, rien dans le domaine littéraire et linguistique n'existant per se, sans être perçu. Les analyses paulhaniennes ne consistent d'ailleurs jamais en autre chose qu'en des indications d'interprétations possibles, laissant de côté toute étude trop formelle, et mêlant toujours la question de la production à celle de la réception. Il s'intéresse donc plus aux emplois possibles qu'à la nature d'un fait d'expression.

Selon Paulhan, si les rhétoriques nous offrent un vocabulaire et une nomenclature, elles ne s'interrogent toutefois jamais sur l'origine du sens des figures. À travers la théorie paulhanienne de la figure émerge donc une réflexion qui dépasse largement la question tropologique traditionnelle telle qu'on peut la retrouver chez un Dumarsais ou un Fontanier. En effet, si l'on en est peu à peu venu à considérer la figure seulement 
comme un «ornement»du discours, elle constitue au contraire pour Paulhan une clé qui lui permet d'interroger les liens du langage à la pensée en tant qu'ils président à l'écriture et à la lecture, apparaissant de manière privilégiée en littérature. Il aborde en effet le langage en prolongeant les études des psychologues que Théodule Ribot a inspirés, dont Frédéric Paulhan, père de Jean Paulhan et notamment auteur d'un essai intitulé La double fonction du langage (1929). Pour cette doxa philosophique et psychologique du tournant $\mathrm{du} \mathrm{xx}^{\mathrm{e}}$ siècle, qu'on retrouve d'ailleurs dans le Traité de stylistique (1909) de Charles Bally - renouant lui aussi avec l'ancienne rhétorique, avec sa notion de style - l'activité linguistique doit être abordée en tant que reflet de la pensée et moyen d'aborder et de réfléchir sur la cognition humaine, comme chez Lévy Bruhl. Loin d'être seulement une réserve de formes ou une grille de lecture disponibles, les figures constituent chez Paulhan un phénomène où il est difficile de distinguer l'artifice rhétorique - si décrié par la modernité - de l'expression sincère, critère moderne de valeur littéraire depuis le romantisme. Quand John Bender et David Wellbery dressent la liste des conditions historiques du déclin de la rhétorique à l'époque contemporaine dans The Ends of Rhetoric (1990), l'une des conditions de ce déclin est d'ailleurs l'«exigence d'originalité, d'individualité, et de subjectivité du discours littéraire ${ }^{29} »$. Or cette critique et son principe moderne d'originalité se révèlent affublés par Paulhan du qualificatif de terroristes.

Si la tradition n'a cessé de répertorier les figures en groupes selon diverses nomenclatures et configurations, la manière dont Paulhan les classe repose sur l'opposition, banale dans les traités de rhétorique, entre figures de mots et figures de pensée. D'une manière assez sommaire, il explique : «On appelle figure (disent les rhétoriqueurs) tout ornement du langage ${ }^{30} »$. Ce faisant, il développe le principe rhétorique de l'electio, selon lequel il existerait toujours une expression propre ou naturelle et une expression figurée entre lesquelles on pourrait librement choisir. Or cette idée repose sur une conception ancienne du langage pour laquelle « il y a une base nue, un niveau propre, un état normal de la communication, à partir duquel on peut élaborer une expression plus compliquée, ornée, douée d'une distance plus ou moins grande par rapport au sol originel ${ }^{31} »$. De sorte que, d'après Barthes,

[...] récupérer la rhétorique, c'est fatalement croire à l'existence d'un écart entre deux états de langage ; inversement condamner la rhétorique se fait toujours au nom d'un refus de la hiérarchie des langages entre lesquels on n'admet qu'une « hiérarchie fluctuante » et non fixe, fondée en nature ${ }^{32}$.

Paulhan précise d'ailleurs que les expressions figurées constituent « une suite de cas privilégiés, où affleurent les rapports de la langue à l'esprit ${ }^{33} »-$ une expression figurée devant être identifiée comme telle pour exister dans l'esprit d'un sujet.

Le traité de Paulhan fait alterner des chapitres portant sur des types de figures qu'il décrit et illustre au moyen d'exemples forgés de toutes pièces ou de citations d'auteurs contemporains, avec des chapitres de « remarques » qui opèrent un retour réflexif sur les chapitres descriptifs - macrostructure d'ailleurs reprise par Michel Charles dans sa Rhétorique de la lecture. Or, dans ses remarques, Paulhan ne cesse d'exposer l'ambiguïté du classement des figures qu'on trouve dans les rhétoriques en général, mais aussi dans la sienne. Ses conclusions aboutissent toujours au même constat: le repérage et le classement des figures dépendent de la lecture qu'on en fait autant que du cotexte où se trouvent ces figures, ce à quoi s'ajoute le fait qu'aucune manière d'écrire ne peut programmer une seule et unique lecture. Tout est question, pour Paulhan, d'attention. Il écrit ainsi, à la fin d'une première salve de remarques où il tente de montrer que l'effet 
équivoque d'une figure de pensée peut être effacé ou fortifié par le contexte de son utilisation : «On citerait aisément mille autres exemples, où l'expression dans son jeu semble échapper aux classes et catégories rhétoriciennes, à la fois par le haut et par le bas - par l'extrême conscience comme par l'extrême inconscience ${ }^{34}$ ». Une figure requiert donc, pour atteindre son plein fonctionnement, l'attention d'un sujet qui sait la lire de manière non littérale.

Paulhan renforce ce constat en abordant la question des figures de langage ${ }^{35}$ (dans lesquelles il regroupe les figures de construction et les tropes). Dans ce cadre, le traitement que fait Paulhan de la métaphore est particulièrement intéressant. Pour lui, "le type même du trope se trouve être ainsi la métaphore - où la comparaison [...] se trouve resserrée, concentrée, réduite à un seul $\operatorname{mot}^{36} »$. Cependant, il existe des cas où, selon lui, l'on hésite entre comparaison et métaphore :

De l'une à l'autre cependant, dans le même texte, courent quelques phrases dont on peut douter, à première vue, s'il faut les tenir pour images ou pour comparaisons :

Les places longues de la ville, avec leurs mosaïques noires ondulées, semblaient arrosées d'encre fraîche

(voilà qui paraît retenu, par ce «semblaient ", dans l'ordre de la comparaison, au lieu que :

Des hommes nègres, armés de tuyaux en caoutchouc, flattaient de la main les palmiers les moins bouffis

glisse déjà vers l'image, que vient encore accentuer - et comme fixer :

L'air brûlait mais léché par des langues de glace $\left.{ }^{37}\right)$.

À partir de là, Paulhan conclut :

[...] il faudrait distinguer entre les figures invisibles, devenues habituelles et comme passées dans la langue [...], et les mêmes figures à l'état surprenant ou sensible [...]. Entre l'une et l'autre catégorie prendraient place nombre d'expressions, qui prêtent au doute et se voient, suivant le cas, tantôt entendues en figure et tantôt en réalité ${ }^{38}$.

Relevons que le critère de distinction entre les figures de rhétorique avancé par Paulhan repose seulement sur la potentielle perception de la figuralité d'une figure par un sujet. Or la comparaison est une figure marquée formellement, à la différence de la métaphore, considérée en tant que figure de mots (selon la typologie paulhanienne, qui ne hiérarchise d'ailleurs pas ces deux types de figure contrairement à la tradition), d'où les doutes de Paulhan sur cette dernière, comme l'explique Michel Charles à son sujet :

Tout se passe donc comme s'il était deux catégories de figures : l'une où la figure peut devenir « catachrèse » tout en restant figure; l'autre où ce curieux processus n'est pas possible. Le rhétoricien trouve quelque réconfort dans le premier cas (figures de pensée), puisqu'il lui est toujours loisible de parler de figure, mais rencontre un choix difficile dans le second (figures de langage), puisqu'il devra «prendre parti » en disant si oui ou non il y a figure. En d'autres termes, dans le premier cas, l'analyse rhétorique se suffit; dans le second, elle renvoie à un acte proprement critique ${ }^{39}$.

Comme l'écrit Paulhan : « La métaphore n'arrête pas d'apparaître et de disparaître ; elle est à n'en pas finir ${ }^{40} »$. Elle constitue de ce fait un cas privilégié pour aborder la question de l'attention et de l'interprétation dans le processus de lecture.

Dans un autre texte ${ }^{41}$, Paulhan analyse avec la même optique la querelle de l'image dans la poésie surréaliste. Il rappelle, en des termes qui lui sont propres, que le critique Jean Prévost condamne les images poétiques des œuvres surréalistes, quand André Breton les admire: "Les images, dit l'un, sont détestables parce qu'elles ne sont que jeux de mots et que l'auteur se soumet au langage. Les images sont admirables, dit l'autre, parce qu'elles témoignent que l'écrivain s'est une fois pour toutes libéré du langage ${ }^{42}$ ». 
Autant dire que de l'image au cliché, il n'y a pour Paulhan qu'un changement de point de vue. Dans son chapitre sur Paulhan, Michel Charles évoque lui aussi ce lien entre efficacité rhétorique et contexte de réception lorsqu'il écrit qu'«il n’y a pas loin du proverbe "creux" à la réplique heureuse ${ }^{43}$ ". Il s'agit donc pour Paulhan de juger de la fraîcheur sémantique d'une figure, soit de l'éclat de son sens à la lecture, plus que de sa morphologie - ce en quoi les rhétoriques traditionnelles se révèleraient incomplètes ${ }^{44}$, même si Michel Charles précise que, sur ce point, Paulhan retrouve en fait une "problématique attestée par la tradition ${ }^{45}$ " comme en témoigne le discours de Fontanier sur la catachrèse dans Les Figures $d u$ discours. Précisons malgré tout à ce propos que Paulhan ne dispose pas encore des savoirs linguistiques sur le figement entre autres développés dans les années $1970-1980^{46}$, notamment d'un point de vue lexico-syntaxique.

Paulhan bute ainsi sur une tension qui traverse toute l'histoire de la rhétorique. En effet, la rhétorique a toujours constitué à la fois une méthode pour concevoir et exécuter un discours, tout en étant un outil permettant de juger un texte - en se mêlant par exemple, en France, à la poétique notamment par le biais de la lexis ${ }^{47}$. Or c'est cette fine frontière que veut mettre en évidence le propos de Paulhan, en distinguant notamment deux points de vue possibles sur un texte : le point de vue de celui qui le lit en écrivain qui connaît toutes les techniques et les règles d'écriture et le point de vue du pur lecteur, de celui qui ne s'attarde pas sur la forme. La lecture idéale pour Paulhan est en effet celle où le langage et la construction se font oublier, où le lecteur fait corps avec les mots au point d'oublier qu'il les lit. Il explique en ce sens que c'est ce critère de la visibilité des figures qui légitime la différence entre figures de mots et de pensée :

[...] la figure de pensée se trouve assurée d'une certaine constance de l'attention. Les " louanges, tirées au petit bonheur, ainsi que des boules de loto d'un sac », voilà qui est, et a toutes chances de demeurer - et l'on ne voit même pas comment elle pourrait cesser d'être - une comparaison (plus ou moins frappante et personnelle, plus ou moins plaisante, il va sans dire). Mais la figure de mots, au contraire, serait à ce compte la figure capable par l'excellence de l'un ou l'autre état extrême et apte aussi bien, à tout instant, à n'être qu'un mot entre tant d'autres, au service de l'événement qu'il s'agit d'exprimer, qu'à constituer l'événement même et la chose que les mots voisins reçoivent tout aussitôt fonction d'exprimer. Bref, c'est une expression dont le sens ni la portée ne sont jamais donnés à coup sûr ${ }^{48}$.

La figure de pensée frappe donc tout lecteur (à l'exemple de la comparaison) qu'il soit ou non critique, alors que la figure de mots n'apparaît en tant que figure qu'à celui qui adopte une lecture critique, savante et formaliste et peut sinon rester invisible à la lecture. Paulhan donne pour preuve de la multiple possibilité de lire un texte le problème de la traduction, qui le taraude depuis son séjour à Madagascar en 1908 :

Un texte en langue étrangère que nous traduisons nous paraît, en règle générale, bien plus imagé qu'il ne l'était pour son auteur. Dans notre langue même, un mot désuet, un terme d'argot suffit à l'erreur. Il n'est même pas nécessaire d'aller chercher l'argot. Quand Giraudoux écrit, entre cent métaphores évidentes : Un nuage voilait le soleil

fait-il une image, a-t-il songé à voile ? Ou bien dit-il simplement voilait comme il eût dit cachait ou dissimulait. L'erreur est à tout instant permise ${ }^{49}$.

Tout est donc question de point de vue et d'effet, sans qu'il s'agisse pour autant de défendre un relativisme absolu. La question est au fond celle de la perception, figée ou non, de la phraséologie, mais il manque à Paulhan - on l'a dit - les notions de figement linguistique qui vont se développer plus tard. Pour lui, un énoncé peut changer de statut si on le considère du point de vue de l'auteur ou du lecteur et du critique, c'est-à- 
dire soit du point de vue de celui qui lit ou écrit avec sincérité (quitte à retrouver sans le vouloir des lieux communs littéraires), soit de celui qui juge au moyen d'une grille de lecture rhétorique. Or c'est bien ce qui ressort de la critique de Valéry que fait Paulhan :

Si l'on en croit Paul Valéry, l'écrivain ne pense jamais fuir la rhétorique, que pour mieux la retrouver. Toute œuvre littéraire est un faux qui nous touche d'autant plus - qui nous paraît d'autant plus spontané - qu'il est plus concerté, plus conscient, plus artificieusement combiné. C'est de quoi Valéry donne comme exemples la « servante au grand coeur » de Baudelaire (pure fabrication, dit-il, n'y ayant jamais eu de servante), le «soleil noir» de Hugo (ce non-sens, doué d'une résonance magnifique), la "majesté des souffrances humaines» de Vigny (beaux mots, parfaite sottise) et maints passages de Stendhal ou Pascal où nous étions accoutumés de voir le type même de l'inspiration et du primesaut. D'où il suit clairement que l'écrivain, loin de redouter la conscience accrue que donne aux tours et figures de langage le souci rhétorique, doit encore aggraver cette conscience, et l'étendre dans la mesure du possible ${ }^{50}$.

Mais Paulhan trouve les exemples de Valéry peu convaincants. Si ce qui paraît spontané se révèle soigneusement concerté pour Valéry, et comme prévu par l'auteur, ce dernier serait en fait sujet à une illusion propre aux critiques tenants de la rhétorique, l'intention auctoriale demeurant dans les faits à jamais mystérieuse, de même que la plupart des impressions de lecture des lecteurs ordinaires. Paulhan précise d'ailleurs :

[...] les faits qu'il évoque [sont] controuvés : la servante a bien existé; le «soleil noir » (qui ne peut guère choquer qu'un savant) flatte certain manichéisme du sens commun: au demeurant, fort courant en littérature, de l'«obscure clarté » de Corneille au «soleil noir » de Swift et de Nerval, et qui semble plein de sens. Vigny n'entend désigner par « souffrances humaines » que les douleurs morales. La sincérité de Stendhal ou de Pascal, si même les mots dont ils usent donnent le sentiment de l'astuce et de la combinaison, ne parait pas niable ${ }^{51}$.

Plusieurs lectures sont donc possibles, de sorte que la figure de rhétorique ne parait telle qu'à celui qui la connaît et la recherche - telle est d'ailleurs la condition même de son efficacité dans le système rhétorique, où l'idéal de «naturel » règne au moins depuis la Rhétorique d'Aristote, selon qui, dans un discours réussi, l'artifice ne se remarque pas et reste dissimulé, même si le naturel reste construit. Le propos de Paulhan met ainsi en évidence l'un des problèmes que comporte tout acte d'interprétation d'un énoncé selon les grilles de la rhétorique. C'est en ce sens que Barthes dira de la distinction entre figures de mots et figures de pensée qu'elle est "mentaliste», puisqu'elle "met en scène des signifiés et des signifiants, les uns pouvant exister sans les autres ${ }^{52} »$. Dans son traité, Paulhan divise d'ailleurs les figures de pensée en fonction des facultés : raison, imagination, passion, etc.

Il serait ainsi bon d'appuyer toute analyse critique sur un certain nombre d'indices sans quoi on risquerait de voir un artifice rhétorique là où l'auteur a écrit avec la plus grande sincérité possible. Parallèlement, il faudrait que l'écrivain mette en évidence et exhibe son usage des figures de rhétorique en tant que figures - comme le faisaient les surréalistes en détournant par exemple explicitement des lieux communs et en soulignant ce détournement par ce que la linguiste Jaqueline Authier-Revuz nomme des formes d'hétérogénéité montrée. Pour Paulhan, l'écriture doit trouver le moyen de mettre en évidence son «traitement rhétorique » du langage. Même si Paulhan ne développe pas outre mesure cette question, on peut imaginer qu'une simple mise en italique pourrait suffire ${ }^{53}$ à une mise en évidence. Mais sur ce point, le propos théorique de Paulhan est, comme souvent, trop léger pour pouvoir vraiment être abouti. 
Quoi qu'il en soit, se pose ainsi pour Paulhan la question du statut de la rhétorique au $\mathrm{xx}^{\mathrm{e}}$ siècle. Est-elle l'instrument d'une lecture critique du texte littéraire ou un savoir permettant d'écrire ? Le Terroriste, en effet, a une lecture rhétorique des textes - qu'il interprète comme le résultat de l'application de règles et de figures préfabriquées ${ }^{54}$ - et cette lecture lui fait rejeter la langue littéraire et la littérature avec elle, alors que pour le Rhétoriqueur la règle ou la figure sert l'écrivain, le libère du souci de l'expression et l'exalte. Comme l'écrira Queneau plus tard: "Le classique qui écrit sa tragédie en observant un certain nombre de règles qu'il connaît est plus libre que le poète qui écrit ce qui lui passe par la tête et qui est l'esclave d'autres règles qu'il ignore ${ }^{55} »$. Autrement dit, selon Paulhan, le risque de la rhétorique est celui de prendre son impression de lecture savante pour une réalité, alors que «c'est la lecture savante, qui perçoit dès l'abord le cliché ${ }^{56}$ ». Pour Paulhan, il faut être conscient que l'on juge un texte sur un effet ou une impression plus que sur un aspect purement formel lorsqu'on pratique une lecture rhétorique d'un texte littéraire: "ainsi l'écrivain qui relit ses œuvres de jeunesse est régulièrement frappé de leur caractère artificiel et verbal. Mais lorsqu'il écrivait, c'était au contraire de leur spontanéité, de leur jaillissement qu'il s'enchantait ${ }^{57}$ ». Remettre la question de la rhétorique au centre des discussions critiques, c'est donc mettre en évidence la relativité de toute analyse critique d'un texte et réinscrire le jugement dans une perspective en partie phénoménologique. Paulhan précise d'ailleurs qu'il faut renoncer, "si le naturel n'est que l'une des formes de la figure, à distinguer le style figuré du style nature ${ }^{58}$ ». Comme chez Nietzsche ${ }^{59}$, c'est l'ensemble du langage qui semble figuré à Paulhan, de sorte que le «sens propre » est composé de figures dont la nature figurée a été oubliée. On retrouve là le thème de l'attention critique. Les tropes et les figures seraient ainsi «des structures fondamentales du langage, et non des exceptions ou des distorsions ${ }^{60}$ ». Paulhan cite à ce propos l'historien de la littérature Eugène Géruzez qui aurait écrit : «Les figures sont dans le langage commun ce que sont, dans les champs et les jardins, les fleurs que l'on reconnait ${ }^{61}$ ». Et Paulhan de préciser: "C'est dire que les figures ont pour seule caractéristique les réflexions et l'enquête que poursuivent à leur propos les rhétoriqueurs - soit la division des figures de pensée d'avec les figures de langage ${ }^{62}[. .]$.$» . Il invite donc le critique à rechercher ce qu'il appelle des$ " paramètres », soit des indices permettant une lecture rhétorique d'un énoncé, « telles que la citation, l'expression toute faite, la locution proverbiale, le proverbe ${ }^{63} »$. Il faudrait, autrement dit, repérer les indices de mise en évidence d'un fonctionnement rhétorique d'un segment textuel, le calembour ou le jeu de mots pouvant par exemple fonctionner comme des paramètres programmant une lecture rhétorique ${ }^{64}$, car il est évident que "toute décision de forme engage le sens du texte ${ }^{65}$ ", sans toutefois entièrement le prédéterminer. Paulhan résume donc sa conception de la figure de cette manière :

[...] la figure est une expression sensationnelle ; qui obtient plus d'effet, qui frappe plus que toute autre expression, et plus que tout autre peut emporter l'émoi, l'adhésion du lecteur (ou de l'auditeur) [mais] cette expression donne régulièrement à douter si elle est naturelle ou concertée, spontanée ou artificielle : si l'arrangement et la combinaison des mots y prennent le pas sur la pensée, ou la pensée sur les mots ${ }^{66}$.

Ainsi abordée dans la perspective de l'analyse de texte, la figure, dit Paulhan, apprend donc à lire et oblige à relire. De cette manière, la rhétorique devient un " art de lire ${ }^{67}$ ", au sein duquel le discours littéraire est appréhendé en tant qu'effet en partie 
indépendant d'une quelconque volonté d'auteur et qui s'inscrit dans la relation d'un sujet à une œuvre de langage autant que de style.

Or, dans un geste d'appropriation herméneutique et de détournement partiel, Michel Charles reprend cette analyse là où Paulhan s'arrête ${ }^{68}$, mais en esquivant la délicate question de l'intentionnalité d'auteur et de la naturalisation du sens, pour revendiquer pleinement le geste critique que constitue une "lecture rhétorique ». En déplaçant légèrement la focale, il soutient en effet que "la littérature est un effet rhétorique spécifique ${ }^{69}$ ", puisque tout texte, si on l'analyse rhétoriquement, détermine en partie sa lecture en indiquant la manière dont il doit être lu. À la lecture du chapitre qu'il consacre à Paulhan et à la question de la lecture des figures de rhétorique, on comprend à quel point les écrits de Paulhan ont compté pour la conception de la Rhétorique de la lecture. Pour Michel Charles, la " "justesse" du poème" tient par exemple « dans une large mesure, à l'attitude de lecture qu'il nous impose $\mathrm{e}^{70} »$ : soit aux types de procédés poético-rhétoriques que le texte poétique met lui-même en évidence et qui s'imposent au lecteur. En effet, « la "mise en condition" du lecteur ${ }^{71}$ " - notion connexe à ce que Paulhan appelait "paramètres» - est essentielle à la lecture et une "rhétorique de l'effet» telle que la développe Michel Charles tend à décrire «les procédures textuelles qui rendent [d]es lectures possibles $^{72} »$. Ce dernier dépasse donc la problématique paulhanienne d'une phénoménologie des figures de rhétorique et l'élargit à une théorie de la lecture.

Le cas des figures n'en demeure pas moins intéressant : elles constituent, chez Michel Charles, autant de questions que l'on peut poser à un texte : la "flamme" dans un poème est-elle bien une métaphore de "l'amour " ? Et sinon, quel est son sens ${ }^{73}$ ? La lecture devient donc rhétoricienne : « une figure, c'est une décision à prendre ${ }^{74}$ ». Ainsi, «métaphore, synecdoque, ironie, et tout ce qu'on voudra, ne sont pas des descriptions de procédés d'écriture : ce sont différents types de questions à poser et différents parcours à effectuer ${ }^{75}$ ». Si la rhétorique peut bien évidemment toujours constituer une grille de production d'un texte, elle devient donc chez Michel Charles, à la suite de Paulhan, un code, une grille de lecture critique qui doit déterminer comment le texte se donne à lire (et non pas simplement décrire des procédés d'écriture d'un point de vue extérieur selon l'optique normative des rhétoriques classiques). Michel Charles l'explique ainsi :

La rhétorique est en effet une théorie du discours, mais, si je puis dire, du discours en tant qu'il est reçu, ou à recevoir, de telle ou telle manière. Disons une théorie du discours comme « effet ». Or, c'est la lecture que nous en pratiquons qui fait qu'il y a quelque chose de tel que la littérature ${ }^{76}$.

La redécouverte de la rhétorique effectuée par Paulhan s'accompagne d'un changement de perspective, et ce, parce qu'elle pose les figures au fondement de sa théorie, comme l'explique Michel Charles: "la figure, hypothèse de lecture; la rhétorique, art de lire $^{77}$ ». Ce geste mène ainsi jusqu'à Michel Charles, pour qui la rhétorique n'ouvre plus seulement sur une intuitive phénoménologie de la réception, mais devient " délibérément un "art de lire", envisageant le discours en fonction des interprétations possibles, et les mettant en perspective sur une inconnue : la lecture à venir ${ }^{78}$ ». C'est que, lorsque Michel Charles prend la plume, la réflexion sur le langage a bien évolué depuis l'époque de Paulhan. 


\section{En conclusion : une « rhétorique » de circonstance, pour en revenir aux fleurs}

On l'a dit, Paulhan tente de transformer la rhétorique classique en quelque chose de neuf. Il le fait pour la simple et bonne raison qu'il pense devoir répondre au régime littéraire de la Terreur. Pour lui, la Terreur repose sur un discours commun à un certain nombre d'écrivains, mais aussi de critiques littéraires :

Tantôt, en effet, le critique tient que le fond entraîne la forme, et qu'on écrit toujours assez bien quand on a quelque chose à dire. Tantôt que la forme guide le fond, et que la pensée vient toujours habiter la demeure qu'un soin suffisant lui a ménagée. Ainsi, les uns admettent - avec tous les Classiques, et Rhétoriqueurs - que l'esprit procède du langage, et qu'une œuvre accomplie suivant les règles et lois de l'expression enferme toujours assez de pensée. Mais les autres - avec les adversaires de la Rhétorique : Romantiques, Terroristes - que le langage n'est bon qu'à opprimer la pensée, et que l'œuvre rhétoricienne, trop soumise aux règles, a le grand tort d'être fausse, abstraite et banale ${ }^{79}$.

Il s'agit donc pour Paulhan de proposer une méthode d'analyse de texte alternative, qui renoue avec ce que la littérature peut encore avoir de rhétorique à l'époque contemporaine. Puisque "la figure, comme le cliché, dépend d'une prise de conscience », il faut recourir à une "conscience rhétorique du langage ${ }^{80}$ » complète Michel Charles. La rhétorique fait en effet hésiter le lecteur sur le statut concerté ou non d'une figure dans un texte, raison pour laquelle il faut repérer les indices qui programment une lecture rhétorique et soulignent la figuralité des figures. Or dans son essai Petite préface à toute critique (1951), Paulhan examine les relations possibles entre divers énoncés et leurs différents sens - ce qui est l'enjeu propre d'une lecture rhétorique. Il regrette ce qu'il conçoit comme le passage de la critique moderne à un régime réflexif qui interdit le jugement et délaisse rhétorique et poétique oubliant ainsi, pour reprendre Valéry, que « la littérature est, et ne peut être autre chose qu'une sorte d'extension et d'application de certaines propriétés du Langage ${ }^{81}$ ". L'histoire de la critique littéraire, bien sûr, corrigera ce constat d'oubli. Selon lui néanmoins, la plupart des critiques négligent la possible polysémie d'un énoncé en lui attribuant une signification prédéterminée grâce à une grille de lecture préexistante, sans suffisamment prendre en compte les indications de lecture et d'interprétation que donne le texte littéraire lui-même et la liberté d'interprétation du sujet-lecteur. Et si les critiques le font, ils se révèlent partiaux. Là où un Valéry voit de l'artifice et de la rouerie, on pourrait, dans certains cas, supposer de la spontanéité. Chez Paulhan, où le sens est le plus souvent conjectural et indiciel, la lecture critique devient ainsi une réécriture au service des lectures et des écritures ; chez Michel Charles, elle deviendra une véritable théorie du texte littéraire, ainsi que, de manière plus contemporaine, chez les théoriciens des "textes possibles ", comme Pierre Bayard, Jacques Dubois, Sophie Rabau et Marc Escola. Si tout discours littéraire comporte des figures et une rhétorique - de même que les jardins à l'anglaise contiennent autant de fleurs que les jardins à la française -, encore faut-il que le critique se donne les moyens de les repérer en tant que telles et de comprendre leur fonctionnement en tant qu'éléments d'un texte toujours perçu. Pour la critique de l'attention qui fut celle de Paulhan, il fallait donc savoir être attentif à cette «chose délicate qu'[est] le jeu du langage [qu']on entend ici grincer : ces lieux communs, phrases toutes faites, proverbes et toute cette couche supérieure des mots ${ }^{82} »$. 


\section{NOTES}

1. Il ne la distingue d'ailleurs pas entièrement de la poétique.

2. G. Genette, «Introduction », dans P. Fontanier, Les Figures du discours, Paris, Flammarion, 1977, p 13.

3. On songe notamment à Point du jour (1934) de Breton, Ralentir travaux (1930) d'Éluard ou encore 152 Proverbes mis au goût du jour (1925) d'Éluard et Péret, où « il faut battre le fer pendant qu'il est chaud» se transforme par exemple en «il faut battre sa mère pendant qu'elle est jeune". Plusieurs des travaux du chef de file du surréalisme belge, Paul Nougé, s'inscrivent aussi dans ce cadre.

4. J. Paulhan, « La rhétorique renaît de ses cendres » (1938), CEuvres complètes, t. II, Paris, Cercle du livre précieux, 1966, p. 167.

5. C.-V. Langlois, C. Seignobos, Introduction aux études historiques, Paris, Hachette et C ${ }^{\text {ie }}, 1898$, p. 13.

6. Qui a notamment défendu la rhétorique dans deux articles célèbres: "Théorie du lieu commun » (Revue des deux Mondes, 1880) et " Apologie pour la rhétorique » (Revue littéraire, 1890 : voir l'édition critique de cet article dans l'Atelier du présent numéro, par I. Vidotto).

7. R. Barthes, «L'ancienne rhétorique », Communications, n 16, 1970, p. 207.

8. J. Paulhan, « La rhétorique renaît de ses cendres », op. cit., p. 160.

9. Id., " "Poésies" d'Isidore Ducasse" (1920), Euvres complètes, t. IV, Paris, Cercle du livre précieux, 1966, p. 317.

10. B. Monginot, «Jean Paulhan théoricien », dans C. Barthélemy dir., La Littérature selon Jean Paulhan, Paris, Classiques Garnier, 2014, p. 193.

11. L. Jenny, Je suis la révolution, Histoire d'une métaphore (1830-1975), Paris, Belin, 2008, p. 138.

12. Ibid.

13. J. de La Bruyère, "Des ouvrages de l'Esprit», Les Caractères, Paris, Librairie Générale Française, 2012, p. 155.

14. J. Paulhan, «Les Fleurs de Tarbes ou la Terreur dans les Lettres» (1941), CEuvres complètes, t. II : Les Fleurs de Tarbes, éd. Bernard Baillaud, Paris, Gallimard, 2011, p. 121.

15. Id., « La rhétorique renaît de ses cendres », op. cit., p. 157.

16. R. Barthes, «L'ancienne rhétorique », op. cit., p. 193.

17. L'un des reproches traditionnels faits aux figures est d'obscurcir la pensée à travers des expressions opaques.

18. M. Fumaroli, L'Âge de l'éloquence: Rhétorique et "res literaria» de la Renaissance au seuil de l'époque classique, Genève, Droz, 1980, p. 10.

19. Voir le chapitre «Conscience et inconscience du langage » (M. Charles, Rhétorique de la lecture, Paris, Éditions du Seuil, 1977, p. 97-117).

20. R. Barthes, « L'ancienne rhétorique », op. cit., p. 173.

21. L. Paulhan, «Traité des figures ou La rhétorique décryptée » (1938), Cuvres complètes, t. II, Paris, Cercle du livre précieux, 1966, p. 197.

22. Id., « La rhétorique renaît de ses cendres », op. cit., p. 163.

23. Ibid.

24. Ibid., p. 219.

25. Ibid., p. 195.

26. J. Paulhan, « Traité des figures ou La rhétorique décryptée » (1938), op. cit., p. 201.

27. Ibid., p. 202.

28. Ou figures rhétoriques en ce qu'elles s'opposent aux figures stylistiques, voir sur cette distinction H. Suhamy, Les figures de style, Paris, Presses Universitaires de France, 2016, Chapitre VII, p. 107. 
29. Cité par A. Compagnon, «La rhétorique à la fin du XIX siècle (1875-1900) », dans M. Fumaroli dir., Histoire de la rhétorique dans l'Europe moderne, 1450-1950, Paris, Presses Universitaires de France, 1999, p. 1261.

30. J. Paulhan, «Traité des figures ou La rhétorique décryptée », op. cit., p. 201.

31. R. Barthes, « L'ancienne rhétorique », op. cit., p. 218.

32. Ibid.

33. J. Paulhan, «Traité des figures ou La rhétorique décryptée », op. cit., p. 202.

34. Ibid., p. 212.

35. La typologie et la nomenclature de Paulhan diffèrent d'ailleurs des nomenclatures traditionnelles, car la distinction entre « figures de pensée » et « figures de langage » chez lui ne recouvre pas exactement la distinction traditionnelle entre figures de mots (lexis) et figures de pensée (dianoia).

36. J. Paulhan, "Traité des figures ou La rhétorique décryptée », op. cit., p. 217. Sur la même page, Paulhan décrit les tropes ainsi : «Les rhétoriqueurs ont coutume d'appeler tropes, ou figures de sens (mais disons plutôt de signification, puisque nous en sommes aux mots) diverses figures, caractérisées par le fait qu'un terme, une phrase, une période même y voient leur sens transformé. »

37. Ibid., p. 218. Paulhan cite ici le roman Adorable Clio (1920) de Jean Giraudoux.

38. Ibid., p. 222.

39. M. Charles, Rhétorique de la lecture, op. cit., p. 99.

40. J. Paulhan, «Traité des figures ou La rhétorique décryptée », op. cit., p. 226.

41. Voir Id., Clef de la poésie (1944), Euvres complètes, t. II, Paris, Cercle du livre précieux, 1966, p. 262-267.

42. Ibid., p. 266.

43. Voir M. Charles, Rhétorique de la lecture, op. cit., p. 117.

44. «Voilà une intention, pourtant essentielle au discours qu'elle gouverne, sur laquelle ni l'auteur ni, chose plus étrange, le rhétoriqueur ne songent à nous renseigner » (J. Paulhan, «Traité des figures ou La rhétorique décryptée », op. cit., p. 223).

45. M. Charles, Rhétorique de la lecture, op. cit., p. 98.

46. Voir à ce sujet les travaux de S. Mejri, dont: "Le figement lexical : nouvelles tendances ", Cahiers de lexicologie, $\mathrm{n}^{\circ} 80,2002$, p. 213-225.

47. Voir J. Culler, "Rhétorique, poétique et poésie », Théorie littéraire, trad. Anne Birien, SaintDenis, Presses universitaires de Vincennes, 2016, p. 101-118.

48. J. Paulhan, «Traité des figures ou La rhétorique décryptée », op. cit., p. 226. Les italiques sont de l'auteur.

49. Ibid.

50. J. Paulhan, "Autour des "Fleurs de Tarbes" ", Euvres complètes, t. III : Les Fleurs de Tarbes, éd. B. Baillaud, Paris, Gallimard, 2011, p. 405. Paulhan mentionne ici les analyses valéryennes, tirées de Tel Quel (1941), de « La servante au grand cœur » de Baudelaire, de « l'affreux soleil noir d'où rayonne la nuit » des Contemplations de Hugo et encore de la « majesté des souffrances humaines » de « La maison du berger (III) » d'Alfred de Vigny.

51. Ibid.

52. R. Barthes, «L'ancienne rhétorique », op. cit., p. 219.

53. Voir, à ce sujet, l'étude que Paulhan consacre à Jules Renard, qu'il place sur un piédestal littéraire: J. Paulhan, "Jules Renard », Euvres complètes, t. IV, Paris, Cercle du livre précieux, 1969.

54. On en trouverait un exemple dans l'exercice effectué par Paulhan dans son étude "Énigmes de Perse » (parue en plusieurs livraison dans la Nouvelle Revue Française, de novembre 1962 à mars 1964) où il recense notamment le nombre de figures de style dans un recueil de Saint-John Perse 
afin de montrer le ridicule de ce type de lecture, car il est probable que Perse ait « redécouvert » les figures en écrivant.

55. R. Queneau, Le Voyage en Grèce, Paris, Gallimard, 1973, p. 39.

56. M. Charles, Rhétorique de la lecture, op cit., p. 113.

57. J. Paulhan, Les Fleurs de Tarbes ou la Terreur dans les Lettres, op. cit., p. 160 (en note).

58. Id., " Traité des figures ou La rhétorique décryptée ", op. cit., p. 228.

59. «Les figures rhétoriques, c'est-à-dire l'essence du langage", disait Nietzsche (cité par L. Bellenger, L'expression orale [1979], Paris, Presses Universitaires de France, «Que sais-je ?», 1993, p. 65).

60. J. Culler, « Rhétorique, poétique et poésie », op. cit.

61. Cité par J. Paulhan, "Traité des figures ou La rhétorique décryptée », op. cit., p. 229. Celui-ci condense en fait le paragraphe par lequel E. Géruzez (1799-1865) conclut son exposé sur les figures, dans son Cours de littérature. Rhétorique, poétique, histoire littéraire, « ouvrage approuvé [dès 1841] pour les écoles publiques par le Ministre de l'Instruction publique », Paris, Delalain, 1871 ( $17^{\mathrm{e}}$ édition », la « $32^{\mathrm{e}}$ » étant de 1885), vol. I, p. 194 : « Il nous semble aussi que la connaissance des figures ajoute un charme de plus aux beautés du langage. Dans les champs et les jardins, les fleurs plaisent sans doute à l'ignorant comme au botaniste par leur parfum et l'éclat de leurs couleurs ; mais le naturaliste qui sait leurs noms, qui connaît leur famille, les retrouve comme de vieilles connaissances avec un sentiment qui tient de l'amitié. La rhétorique sera pour ces fleurs du langage qu'on appelle figures, et qui émaillent les entretiens et les livres, ce que la botanique est pour les fleurs, dont la nature et l'art ont fait la plus belle parure des prairies et de nos parterres. »

62. Ibid.

63. Ibid., p. 230.

64. Voir ibid., p. 231.

65. Ch. Noille, «La forme du texte: rhétorique et/ou interprétation», Fabula-LhT, $\mathrm{n}^{\circ} 14$, «Pourquoi l'interprétation?», 2015, http://www.fabula.org/lht/14/noille.html.

66. J. Paulhan, « Traité des figures ou La rhétorique décryptée », op. cit., p. 231-232.

67. M. Charles, Rhétorique de la lecture, op. cit., p. 119.

68. Du fait de ses carences théoriques, d'un penchant pour le mysticisme linguistique, et aussi parce qu'il pose mal le problème en ne considérant la rhétorique que sous l'angle du sens figuré.

69. M. Charles, Rhétorique de la lecture, op. cit., p. 62.

70. Ibid., p. 115.

71. Ibid., p. 62.

72. Ibid., p. 63.

73. Voir ibid., p. 118.

74. P. Malandain, «Compte-rendu de lecture : Michel Charles, Rhétorique de la lecture, Seuil, coll. Poétique, 1977 », Semen, 1, 1983, http://journals.openedition.org/semen/3432.

75. M. Charles, Rhétorique de la lecture, op. cit., p. 118.

76. Ibid., p. 79.

77. Ibid., p. 100.

78. Ibid., p. 211.

79. J. Paulhan, «Lettre à Maurice Nadeau ", Euvres complètes, t. III : Les Fleurs de Tarbes, éd. B. Baillaud, Paris, Gallimard, 2011, p. 401.

80. M. Charles, Rhétorique de la lecture, op. cit., p. 102. C'est Paulhan qui souligne.

81. P. Valéry, "L'enseignement de la poétique au Collège de France ", Euvres Complètes, vol. I, Paris, Gallimard, « Bibliothèque de la Pléiade », 1957, p. 1438.

82. J. Paulhan, «Le Reproche que l'on fait aux lieux communs... », CEuvres complètes, vol. II : L'art de la contradiction, éd. B. Baillaud, Paris, Gallimard, 2009, p. 198. 


\section{AUTEUR}

FRANÇOIS DEMONT

Université de Lausanne 